\title{
Factores incidentes en la creación de una unidad de negocios para graduados
}

\author{
Incident factor in the creation of a business unit for graduates \\ Said Diez Farhat*, María Auxiliadora Vargas Valdiviezo, \\ Priscila Fernández Duque
}

\begin{abstract}
Universidad Católica de Santiago de Guayaquil, Ecuador
\end{abstract}
Recibido el 5 de mayo de 2019; aceptado el 28 de abril de 2020

Disponible en Internet el: 10 de mayo de 2020

\section{Resumen}

Esta investigación tuvo como objetivo cuantificar los factores que inciden en la creación de una unidad de negocio mediante un análisis estructural en los graduados de las carreras y centros de capacitación universitarios en emprendimiento de la ciudad de Guayaquil. La metodología a utilizar en esta investigación fue el método deductivo, el alcance del estudio fue descriptivo y explicativo porque está orientado a identificar los factores que inciden en la creación de una unidad de negocio, mediante un modelo estructural en los graduados de las carreras y centros de capacitación universitarios en emprendimiento. Se demostró que la educación y capacidad de emprendimiento y la transferencia de investigación y desarrollo no tienen una influencia directa y significativa en la capacidad de crear un nuevo negocio.

Código JEL: M10, M13, M16

Palabras clave: Emprendimiento; Normas culturales y sociales; Educación empresarial

\footnotetext{
*Autor para correspondencia Correo electrónico: said.diez@cu.ucsg.edu.ec (S. Diez Farhat).

La revisión por pares es responsabilidad de la Universidad Nacional Autónoma de México. 


\begin{abstract}
The purpose of this paper was to approach the factors that influence the creation of a business unit based on a structural analysis carried out among university and training center graduates in entrepreneurship in Guayaquil, Ecuador. This work adopted a deductive research methodology. The scope of the study was descriptive and explanatory, since this stage led to identify which factors influence on the creation of a business unit by a survey for university and training center graduates in entrepreneurship. The findings indicate that education, entrepreneurship and the transfer of research and development do not have a direct and significant influence on the ability to set up a new business.
\end{abstract}

JEL codes: M10, M13, M16

Keywords: Entrepreneurship; Cultural and social norms; Business education

\title{
Introducción
}

La misión de las carreras profesionales de emprendimiento es fomentar las nuevas unidades de negocio que contribuyan al desarrollo del empleo. Sin embargo recientes investigaciones hallaron relación negativa entre la educación en emprendimiento y la capacidad de generar nuevas unidades de negocio Díaz-Casero, Hernández-Mogollón y Roldán (2012). Recientes investigaciones han aportado de manera específica en cuanto a la variable latente entorno con nuevo conocimiento con respecto a los factores que inciden en la creación de un nuevo negocio. Para Díaz-Casero et al. (2012) los factores ambientales o del entorno que influyen en la creación de un nuevo negocio son: (a) la educación y capacitación empresarial, (b) la transferencia de la investigación y desarrollo; y (c) las normas culturales y sociales. Sus hallazgos demostraron el rol excepcional de las normas culturales y sociales en la capacidad de generar nuevos negocios. Sin embargo, estos autores concluyeron que la educación y capacitación en la creación de nuevos negocios no influye significativamente en dicha creación (no está asociada de manera positiva con la creación de nuevos negocios) sino que más bien es un facilitador indirecto a través de la normas culturales y sociales de la población.

Emprender en un nuevo negocio es un proceso de creación, expansión y consolidación de empresas fundamental para el desarrollo económico porque genera fuentes de trabajo (Schumpeter, 1934). Comprender este fenómeno es de interés para los gobiernos (que necesitan aumentar tasas de empleo), los individuos que tienen la motivación de crear su propio negocio (en búsqueda de beneficios económicos) e instituciones dedicadas a la enseñanza y capacitación sobre este tema (en pro de formar profesionales capaces de crear nuevas empresas competitivas). 
En Latinoamérica existen carreras y centros de capacitación universitarios dedicados a la enseñanza de cómo ser emprendedor. Entre los principales objetivos de este tipo de educación son fomentar el espíritu emprendedor y formar generadores de empleo a través de la creación de nuevos negocios. Por lo tanto, la novedad y originalidad de esta investigación radica en testear el modelo de Díaz-Casero et al. (2012) quienes demostraron lo contrario: que más bien capacitar y enseñar sobre cómo crear nuevos negocios no influye en la generación de nuevas unidades de negocio. Con los resultados que se obtengan dada la población escogida se podrá generar un aporte científico en el área de los negocios, específicamente en el emprendimiento. Adicionalmente, se beneficiará a la sociedad con conocimiento sobre los factores subyacentes en la creación de las nuevas unidades de negocio y ayudará a los gobiernos a implementar políticas para fomentar la generación de fuentes de empleo.

\section{Marco Teórico}

En el trabajo seminal de Gartner (1985), explicó en su revisión de la literatura el fenómeno de la creación de nuevos negocios. Para explicar este fenómeno, propuso que la creación de un nuevo negocio integra cuatro perspectivas: (a) las características del individuo que inicia el negocio, (b) la organización que este individuo crea, (c) el entorno que rodea al nuevo negocio y $(d)$ el proceso por medio del cual se inicia el nuevo negocio. Con respecto al individuo, se concluyó que las características psicológicas que influyen en el individuo que decide comenzar un negocio son: (a) necesidad de logros, (b) locus de control, (c) propensión al riesgo, (d) satisfacción laboral, (e) experiencia previa de trabajo, (f) padres emprendedores, (g) edad y (h) nivel de educación.

Por otro lado, Gartner (1985) determinó que el proceso de empezar un nuevo negocio radica en ejecutar ciertas actividades. El nuevo empresario detecta: (a) una oportunidad de negocio, (b) acumula recursos, (c) innova y produce el producto o servicio, (d) responde al gobierno y a la sociedad (Cole, 1965; Schumpeter, 1934). Sin embargo, otro factor que se consideró es el entorno en el que se va a desarrollar el nuevo negocio. El entorno está determinado por: (a) disponibilidad de fondos para financiamiento, (b) el expertise de quien emprende, (c) personal calificado, (d) accesibilidad de los proveedores, (e) accesibilidad a clientes o nuevos mercados, (f) influencias gubernamentales, (g) disponibilidad de terrenos o instalaciones, (h) acceso a transporte, (i) actitud de la población del área (j) disponibilidad de servicios de apoyo y $(\mathrm{k})$ condiciones de vida.

Lo anterior los llevó a deducir que no necesariamente un sistema de educación y capacitación en emprendimiento contiene todas las herramientas a fin de influir en la creación de nuevas organizaciones. Aunque no exista una asociación positiva entre la educación en emprendimiento y la creación de nuevos negocios; al parecer la educación si afecta de manera 
indirecta a través de los valores culturales y sociales porque estos estimulan la motivación de las personas para crear un negocio. También demostraron que la transferencia de investigación-desarrollo influye de manera positiva y significativa en la creación de un nuevo negocio (Díaz-Casero et al. 2012). Pero para validar, expandir, sintetizar y generalizar lo expuesto por Díaz-Casero et al. (2012), los autores sugirieron que es necesario replicar el modelo de ecuaciones estructurales en otros países y testearlo utilizando una muestra de emprendedores para llevar a cabo un análisis a nivel individual para cuantificar la incidencia de cada factor en la creación de una unidad de negocio.

Una de las últimas investigaciones sobre esta temática realizada por Hundt y Sternberg (2016) concluyó que el contexto nacional y regional impactan de manera significativa en la decisión de abrir un negocio y que las características de nivel individual ejerce influencia general, pero dicha influencia no se mantiene en el tiempo por los distintos factores en los que se ve involucrado el futuro emprendedor, como el nivel de ambición que este tenga. En el Ecuador existen carreras y centros de capacitación universitarios dedicados a la enseñanza de cómo ser emprendedor. Entre los principales objetivos de este tipo de educación son fomentar el espíritu emprendedor y formar generadores de empleo a través de la creación de nuevos negocios.

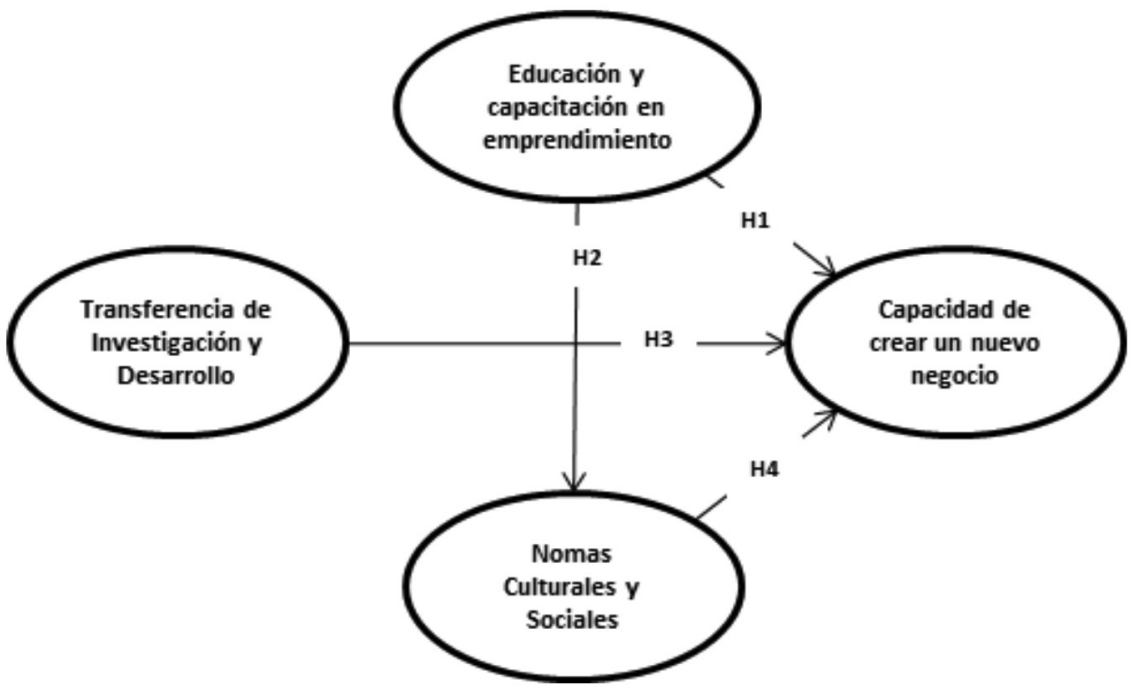

Figura 1. Modelo de la investigación propuesta. Tomado de "A structural model of the antecedents to entrepreneurial capacity," por J. Diaz-Casero, R. Hernández y J. Roldan, 2012, International Small Business Journal, 30(8), 850-872 
A continuación se detalla la revisión de la literatura que sirvió como base teórica para esta investigación.

En el campo empresarial es necesario estudiar la relación que existe entre la educación y el entrenamiento con el emprendimiento. (Henry, Hill, \& Leitch, 2005). Se ha hablado de la relación de la educación con el emprendimiento (Gibb \& Hannon, 2006) puesto que se entiende que las universidades pueden influir en el deseo del estudiante de crear una empresa. Sin embargo, Gibb y Hannon (2006) muestran que el emprendimiento no solo requiere de una adecuada educación superior, sino compromiso con la mejora continua. Un título universitario en una carrera administrativa ya no es suficiente para asegurar que un individuo dure en un trabajo, solo otorga un acceso inicial al campo laboral (Politis et al., 2012; Rovayo, 2013). La educación no asegura un éxito, pero si te da el mapa por donde puedes aumentar la tasa de éxito en cualquier emprendimiento. (Robinson \& Sexton, 1994; Rothaermel et al., 2007).

Actualmente, el ambiente económico global está compuesto por los adelantos tecnológicos y el surgimiento de nuevos mercados a nivel mundial. Por lo tanto, es aparente que un emprendedor tenga necesidad de habilidades de emprendimiento para poder tratar con los retos de la vida y el futuro incierto independientemente de la carrera profesional que una persona escoja o de sus circunstancias, aprender emprendimiento le permitirá aprender a ser innovador en la resolución de problemas, adaptarse a los cambios y a ser más autosuficiente (Henry et al., 2005; Sesen, 2013).

Levie y Autio (2008) explicaron que se espera que la Educación y Capacitación en Emprendimiento (EET) fomente la oferta de emprendimientos. Uno de los mecanismos para fomentar el emprendimiento con EET es a través de la dotación de habilidades instrumentales necesarias para iniciar y hacer crecer una nueva empresa (Honig, 2004). Otro mecanismo es a través de la mejora de la capacidad cognitiva de los individuos para gestionar complejidades involucradas en el reconocimiento y evaluación de oportunidades, así como en la creación y crecimiento de nuevas organizaciones (DeTienne \& Chandler, 2004). Una tercera manera es mediante el efecto de la cultura sobre las actitudes de los estudiantes y las disposiciones de su comportamiento (Peterman \& Kennedy, 2003). Así, el emprendimiento requiere una mezcla de habilidades a fin de eliminar brechas que impidan completar los insumos para el emprendimiento (Levie \& Autio, 2008).

La importancia de la educación en emprendimiento, surge como una gran necesidad debido al aumento del desempleo y subempleo en los países en desarrollo. Los graduados de los centros de estudios permanecen en el desempleo, porque no poseen las habilidades requeridas por los estándares de la industria. Es necesario un sistema educativo basado en las habilidades y el desarrollo de la conciencia empresarial, que se enfoque más en los aspectos prácticos que teóricos, para que existan más individuos que creen nuevas empresas y contribuyan al desarrollo económico del país. Los gobiernos deben garantizar el establecimiento de un buen 
sistema de educación empresarial, con buenos centros de estudios y personal docente calificado para educar y entrenar a los posibles emprendedores y tener fondos disponibles para facilitar el emprendimiento al final del programa (Panigrahi, 2015; Zhao, Seibert, \& Hills, 2005).

La literatura revela una gran relación entre educación, creación de negocios y desempeño empresarial, así como entre educación empresarial y el emprendimiento. Si bien es cierto el mejor indicador para medir el resultado de la educación en emprendimiento es la tasa de creación de nuevos negocios, algunos estudios revelan que esos resultados no se reflejan de inmediato. De acuerdo a los estudios de algunos académicos entre la educación emprendedora y el emprendimiento, revela el impacto positivo de la educación emprendedora, y propone acciones a los gobiernos para que destinen fondos para los programas de educación empresarial y seleccionen los programas más adecuados (Raposo \& Paço, 2011).

Se puede predecir con gran exactitud los distintos comportamientos debido a que la teoría está respaldada por evidencia empírica. La aplicación de esta teoría en la educación empresarial permite entender las intenciones y actitudes de dichos comportamientos de compra o de decisión de manera eficaz ya que brinda información útil. Dicho esto, la evaluación de resultados, la fuerza de creencia, la motivación para cumplir, y el poder percibido de los factores de control permite superar limitaciones de gran escala, pero se presenta un limitante con mucho poder que tiene relación con la ganancia observada entre las medidas globales y creencias (Ajzen, 1991; Parker \& Belghitar, 2006).

La transferencia de la investigación y desarrollo (RDT) tiene un enfoque schumpeteriano, quien enfatizó la importancia del desarrollo tecnológico como generador de oportunidades empresariales (1934). El desarrollo tecnológico crea desbordamientos o spillovers de conocimiento que pueden ser aprovechados para comercializar innovaciones (Nelson \& Winter, 1982). En consecuencia, el emprendimiento desempeña un papel importante para facilitar la explotación de los spillovers del conocimiento.

Según Díaz-Casero et al. la RDT es una variable que estima "en qué medida la investigación y el desarrollo de los países o regiones conducen a nuevas oportunidades comerciales; y distingue si estos están al alcance de los empresarios o no" (2012, p. 852)”. En este aspecto la RDT indica que tan accesible son los nuevos conocimientos y tecnologías para las nuevas empresas y las empresas en desarrollo (Reynolds et al., 2005). Desde esta perspectiva, la innovación (cambio tecnológico) contribuye a la aparición de nuevas empresas sea porque combinan los recursos de una manera distinta o porque crean nuevas funciones de producción (Cooper \& Park, 2008).

Por lo tanto, la RDT es una de las condiciones más significativas para un ambiente emprendedor (facilita el emprendimiento) en especial para los países con altos niveles de competitividad y orientados a la innovación. Sin embargo, para los países cuyas economías se basan en el sector primario la RDT más bien tiene poca significancia. La estructura institucional limita las 
acciones para la creación de empresas en lo referente a creencias, género, legislación mercantil entre otras, lo que condiciona la aparición y desarrollo de nuevas empresas (Díaz-Casero, Urbano, \& Hernández, 2005). Asimismo, la cultura es un factor determinante en la forma en que los individuos afrontan las limitaciones para generar soluciones a corto plazo y la forma de enfrentar las limitaciones formales que las normas sociales pueden llegar a establecer en la eficacia de los empleados en el desarrollo empresarial a largo plazo.

Los seres humanos construyen "modelos mentales" para intentar explicar e interpretar la realidad que los rodea y el ámbito empresarial no es la excepción, de acuerdo a lo expuesto en el enfoque teórico sociocultural que estudia los factores que inciden en la actividad emprendedora (Díaz-Casero et al, 2005). Uno de los aspectos que no se puede quedar aislado en el estudio del emprendimiento es la forma de reaccionar del individuo condicionado por el entorno ya que las normas sociales y la cultural se ponen de manifiesto cuando se trata de analizar el crecimiento económico, la innovación de las empresas y la creación de empleo (Díaz-Casero, Hernández, Sánchez, \& Postigo, 2010). Las sociedades poseen por naturaleza diferentes entornos físicos, los miembros de una sociedad deben adoptar patrones de comportamiento ambientalmente dominantes para lograr el éxito. Estos patrones de conducta ambientalmente dominantes implican la formación de distintos valores culturales en sociedades diferentes; algunos de los cuales inciden en la decisión de crear nuevas empresas (Thornton, Ribeiro-Soriano, \& Urbano, 2011). Consecuentemente, la cultura (a diferencia de los contextos políticos, sociales, tecnológicos o económicos) es determinante para el comportamiento económico y el emprendimiento (Shane, 2000).

El factor ambiental y político puede facilitar o impedir actividades empresariales, así como incentivar o parar el espíritu emprendedor de los estudiantes. En el estudio realizado sorprendió mucho que la actitud hacia el trabajo por cuenta propia es aún más favorable entre los estudiantes de Estados Unidos en comparación a los de Alemania, dando de la misma forma que universidades alemanas con docentes con mayor capacitación y que cuentan con un mejor material de aprendizaje son más baratas que las de Estados Unidos y que no tienen capacidad de seleccionar a sus estudiantes y esto disminuye su espíritu emprendedor (Franke \& Lüthje, 2004). La incubación de negocios da un puente para promover la novedad en los negocios, pero no siempre el resultado de la incubación dará un valor a nuestra idea. Para crear alto valor de incubación deberá haber relaciones fuertes entre socios estratégicos de la misma red. (Hughes, Ireland, \& Morgan, 2007). La iniciativa empresarial muy aparte de involucrar intereses propios une al factor medioambiental, esta incorporación da como resultados beneficios económicos muy altos dando de igual manera mejoras ecológicas que ayudan a la sociedad y al planeta (Shirokova, Osiyevskyy, \& Bogatyreva, 2015). Se trata de formar emprendedores con una misión social concreta introduciendo productos y servicios concretos con el fin de crear alianzas con sectores importantes en la industria. (Lenox \& York, 
2012; Nielsen \& Lassen, 2012).

La literatura concuerda que la cultura es un determinante del comportamiento emprendedor. La cultura se utiliza para referirse al conjunto perdurable de valores de una nación, una región o una organización (George \& Zahra, 2002). Sin embargo, es necesario distinguir entre la cultura nacional general o los valores universales como los medidos por Hofstede (1980) y las creencias o actitudes específicas de acuerdo al contexto con respecto al emprendimiento. Varios estudios empíricos han reportado asociaciones estadísticas entre la cultura y las actividades empresariales. Pero los resultados de los intentos para medir el efecto de la cultura nacional en la actividad empresarial utilizando medidas nacionales de cultura estándar y controles apropiados han sido diversos y mixtos. Lo anterior podría deberse a que las creencias ampliamente compartidas en sociedades determinadas pueden mediar entre los valores culturales y la promulgación de comportamientos específicos (Smith, Peterson, \& Schwartz, 2002). Por lo tanto, se debe diferenciar entre la cultura nacional y la cultura emprendedora y normas sociales (Levie \& Autio, 2008).

La inversión en investigación y desarrollo es muy importante para el crecimiento económico, ya que genera nuevos conocimientos, tecnología y habilidades, además es intensiva en capital humano y este capital es inalienable y portable (Babina \& Howell, 2018). El emprendimiento tecnológico permite transformar la investigación y el potencial de las instituciones científicas en nuevos productos y servicios, que incrementan los beneficios a los consumidores y fortalece el crecimiento económico futuro (Matejun, 2016).

La capacidad emprendedora, conocida en inglés como entrepreneurial capacity (EC), es una herramienta que ayuda al individuo a descubrir las oportunidades del mercado antes de comprometer sus recursos y exponerse al riesgo de crear una empresa. (Clarysse, Tartari, \& Salter, 2011) Estos autores citando a Baron \& Ensley (2006) explicaron que identificar las oportunidades es una de las características que mejor definen al emprendedor y le permiten desarrollar sus habilidades para tener éxito en su emprendimiento.

De acuerdo al Global Entrepreneurship Monitor (GEM) y en concordancia con la literatura, la EC se compone de dos factores: (a) habilidades y (b) motivación. Las habilidades que están implícitas en la capacidad de emprender son (Díaz-Casero et al., 2012):

- Manera de empezar un negocio, es decir si la persona tiene conocimiento sobre cómo fundar una empresa y ponerla en marcha.

- Gestión del negocio, comprende cómo administrar un negocio, conocimiento de temas financieros, mercadeo, ventas y recursos humanos.

- Experiencia en emprendimiento, es decir si el emprendedor ya ha gestado otras empresas.

- Velocidad de reacción ante las oportunidades de un nuevo negocio, implica que 
tan rápido un emprendedor se da cuenta que una oportunidad de mercado (brecha) es un futuro negocio (empresa)

- Organizar los recursos para gestar una empresa, implica si el emprendedor tiene la habilidad de asignar y gestionar los recursos de la empresa tanto financieros como materiales sin causar desperdicio de recursos.

La capacidad emprendedora en la mayoría de los casos ha sido concebida como la solución a la falta de empleo en relación de dependencia, y utilizan su capacidad de creación de negocio como respuesta a esta situación, (Krishnan, Sivramkrishna, \& Warrier, 2017). Sin embargo, esto no está comprobado y es muy importante realizar estudios que permitan determinar cuáles son los factores que inciden en la creación de unidades de negocios (Krishnan et al., 2017).

\section{Método}

Esta investigación se realizó con un diseño no experimental de corte transversal, tomando como unidad de análisis a los graduados en el periodo 2012-2017 de las carreras y centros de capacitación universitarios de la ciudad de Guayaquil. El cuestionario aplicado, es el propuesto por el GEM, lo cual permite utilizar un cuestionario ya validado y la comparación de los resultados con trabajos previos. Para ello, se realizó la traducción de las preguntas del cuestionario original del idioma inglés al idioma español. Para la validación del instrumento de medición, se realizaron entrevistas a expertos para ajustar el contenido de las preguntas a la unidad de análisis. Luego de un proceso de saturación, se logró un cuestionario con validez contextual. Para asegurar que el cuestionario mantenga el sentido original de las preguntas, se realizó una traducción inversa.

Para evitar errores en la aplicación de la encuesta, se realizó una prueba piloto para identificar problemas de entendimiento y errores de formato. Para la realización de la prueba piloto, se aplicó la encuesta a 20 personas que forman parte de la población estudiada. En la aplicación de la prueba piloto, no se encontraron errores de sintaxis ni de forma. Por lo tanto, se consideró que el instrumento de medición ajustado estaba listo para ser aplicado. Para la aplicación del instrumento, se tomó como población de estudio a los graduados de la Carrera de Emprendimiento de la Universidad Católica de Santiago de Guayaquil y el Centro de Emprendedores de la Escuela Superior Politécnica del Litoral. El levantamiento de la encuesta se dio en dos momentos por medio de la difusión del link de la encuesta utilizando la base de datos (869 estudiantes graduados). En un segundo momento, luego de 15 días, se volvió a enviar la encuesta a la misma base de datos para mejorar la tasa de respuesta, obteniendo 102 encuestas válidas, lo cual corresponde a un $11.73 \%$ de éxito de respuesta, lo cual según Hernández, Fernández y Baptista (2014) aseveraron que la baja tasa de respuesta es esperable 
con encuestas en línea.

Una vez tabuladas las respuestas de las encuestas, se procedió a realizar un análisis descriptivo para la identificación de valores faltantes y respuestas atípicas. Debido a que los datos corresponden a variables de intervalo con cinco niveles de respuestas, se analizó el histograma de frecuencias para identificar el comportamiento de los datos e identificar valores faltantes y atípicos. Por medio de este análisis, se encontró que las 27 preguntas del cuestionario, correspondientes a los cinco constructos del estudio no tienen valores atípicos, ni valores faltantes.

Por medio de la inspección visual, de los histogramas de frecuencias se encontró una primera evidencia de la distribución no normal de los datos. La distribución de los datos de las 27 variables observables, mostraron una forma leptocúrtica con asimetría negativa, lo cual se tomó en cuenta al momento de la elección del método de estimación de modelos de ecuaciones estructurales. Se realizó un análisis de la confiabilidad de las escalas de los cinco factores por medio del coeficiente de Alfa de Cronbach. Debido a que se utilizaron escalas sugeridas por la literatura, se recomienda establecer a 0.7 como valor crítico de consistencia interna (Chion \& Charles, 2016). Para el análisis de consistencia interna, se utilizó el paquete estadístico IBM SPSS versión 21.

Tabla 1

Consistencia Interna de los Factores

\begin{tabular}{lcc}
\hline & Número de ítems & Alfa de Cronbach \\
\hline Transferencia de Investigación y Desarrollo & 6 & 0.887 \\
Educación y Capacitación en Emprendimiento & 6 & 0.872 \\
Normas Culturales y Sociales & 5 & 0.822 \\
Habilidades y Capacitación & 5 & 0.904 \\
Motivación & 5 & 0.786 \\
Capacidad para Crear un Nuevo Negocio & 10 & 0.892 \\
\hline
\end{tabular}

Nota: Nivel crítico de consistencia interna 0.7 .

Por medio del análisis del coeficiente de Alfa de Cronbach, se demostró según la Tabla 1 , que las escalas son confiables para el estudio de la capacidad emprendedora para los graduados de la carrera de emprendimiento en la ciudad de Guayaquil. Todos los valores de consistencia interna son mayores al valor crítico de 0.7. Sin embargo, debido a que el estudio relaciona constructos, es necesario evaluar la calidad del modelo por medio de Análisis Factorial Confirmatorio para analizar las cargas factoriales, validez discriminante y validez convergente (Brown, 2015). 
Existen principalmente dos métodos de estimación del Análisis Factorial Confirmatorio (AFC) y el Modelo de Ecuaciones Estructurales (SEM): el primero basado en varianzas y el segundo basado en covarianzas (Ringle, Sarstedt, \& Straub, 2012). El método de estimación más utilizado es el basado en covarianzas, aunque no se realice un análisis de adecuación muestral, es un método de estimación sensible a distribuciones de datos no normales con pequeños tamaños de muestra (Ringle, Sven, \& Becker, 2015). Mínimos Cuadrados Parciales (PLS), es un método de estimación basado en varianzas. Aunque no es considerado un método robusto por no tener la posibilidad de valuación de la calidad de estimación por un único índice de bondad de ajuste, permite obtener conclusiones confiables (Kwong \& Kay, 2013).

Dado la distribución no normal de las variables observables y por el pequeño tamaño de la muestra, el método de estimación para AFC y SEM que mejor se ajusta a los datos es por PLS (Brown, 2015). Se analizó la adecuación muestral por medio de la prueba de Kaiser-Meyer-Olkin (KMO), además de la prueba de esfericidad de Bartlett. Las pruebas en mención sirven para justificar que con los datos levantados se puede realizar un análisis factorial. Según los resultados obtenidos los datos se adecúan para la realización de un análisis factorial con un KMO de 0.889, y un valor de significancia de la prueba de Bartlett $<0.05$. Para conocer si los constructos del presente estudio son reflectivos o formativos, se realizó un análisis de correlación de Pearson, encontrando correlación significativa entre las variables observables de cada factor. Con ello, se realizó la estimación de los modelos de medición por cada factor, asumiendo que corresponden a constructos reflectivos. Para la estimación de los modelos reflectivos de medición, se utilizó PLS como método de estimación por medio del paquete estadístico Smart PLS v. 3.

Se estimaron los modelos de medición por medio del algoritmo PLS, el cual permitió evaluar las cargas factoriales, validez discriminante y la validez convergente por medio del AFC. Por medio del análisis de cargas factoriales, se encontró que solo una de las variables observables (CSN05) tiene una carga por debajo del mínimo aceptable de 0.7 en el factor Normas Culturales y Sociales. Con relación al factor Educación y Capacidad en Emprendimiento, la variable observable EET02 tiene una carga por debajo del mínimo aceptable en la evaluación confirmatoria de una escala. Todas las variables observables del factor Transferencia de Investigación y Desarrollo tuvieron cargas por encima de valor crítico de 0.7.

Por otro lado, las dimensiones del factor Capacidad para Crear un Nuevo Negocio, la dimensión de Habilidades y Capacidades todas las cargas tuvieron niveles aceptables por encima del nivel crítico. Sin embargo, las variables observables M3 y M4 de la dimensión de Motivación tuvieron cargas factoriales por debajo del nivel crítico de 0.7. Además, todas las variables observables del factor Motivación tuvieron cargas factoriales por debajo del nivel crítico en el constructo de segundo orden Capacidad para crear un nuevo negocio. Se encontró que todos los factores tienen valores de las varianzas extraídas medias por encima 
de 0.5 , con lo cual las variables observables explican más de la mitad de la varianza del factor (Garson, 2016). Hasta este momento la evaluación de la validez convergente por medio de las cargas factoriales y los valores de varianzas extraídas medias, sugieren la necesidad de la refinación de constructos por medio de la eliminación de variables observables con bajas cargas factoriales.

El análisis de la validez discriminante se realizó por medio del criterio de Fornell y Larker y en análisis de las cargas cruzadas de las variables observables (Hair, Hult, Ringle, \& Sarstedt, 2014). Por medio del análisis de Fornell y Larker se encontró que todos los constructos son significativamente distintos según estándares estadísticos. Para la evaluación del modelo de ecuaciones estructural por estimación de mínimo cuadrados parciales, se evaluó la capacidad predictiva del modelo por medio del coeficiente de determinación y del efecto tamaño (Garson, 2016). El modelo estimado tuvo un coeficiente de determinación de 0.442 , aunque no hay valor crítico de aceptación en ciencias sociales, por medio de la comparación con trabajos con propósitos similares, se considera aceptable (Ringle et al., 2012).

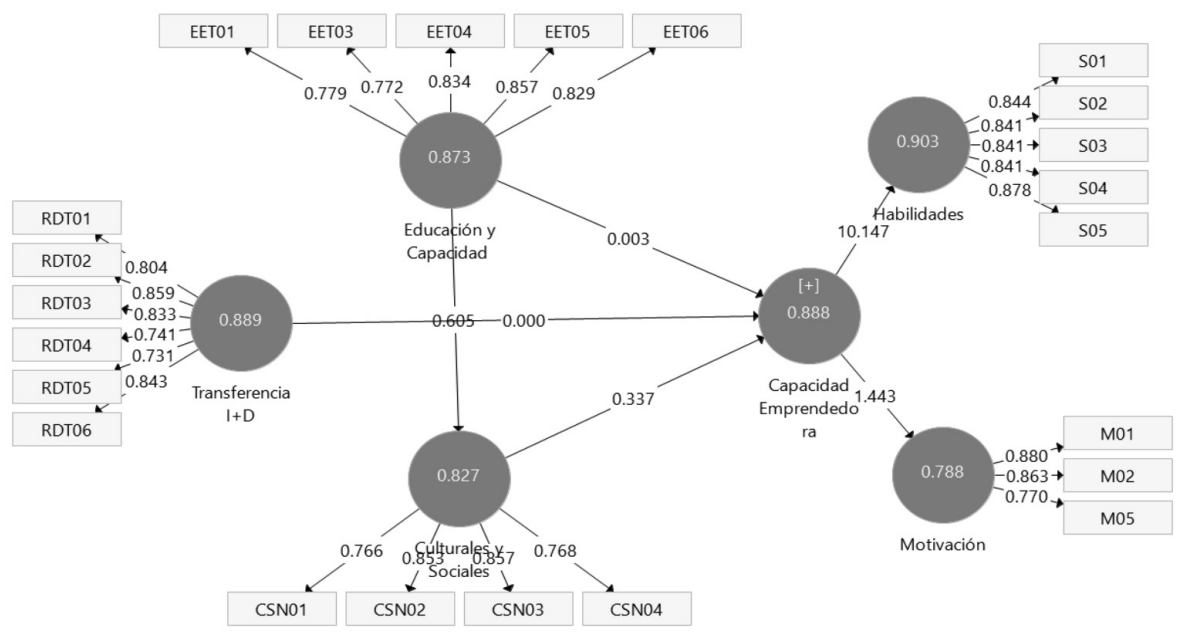

Figura 2. Estimación del Modelo de Medición Ajustado

Sin embargo, los valores de entre los factores explicativos se evidencian una poco significativa capacidad predictiva del factor Transferencia de Investigación y Desarrollo y el factor Educación y Capacitación en la Capacidad Emprendedora. Sin embargo, el factor normas culturales y sociales muestra un nivel medio de predictibilidad en la Capacidad Emprendedora con un valor de efecto tamaño de 0.250. Con estos resultados, se consideró la baja capacidad predictiva del modelo se debería a la incorrecta especificación del factor 
Motivación como constructo reflectivo del factor de segundo orden Capacidad para Crear un Nuevo Negocio. Ante esta situación, se consideró necesario refinar los constructos con bajas cargas factoriales (Harrington, 2009).

Por medio del refinamiento de los constructos por la evaluación de las cargas factoriales, se eliminaron las variables observables con cargas factoriales menores a 0.7. Se sugiere que cuando se prueban escalas sugeridas por la literatura se establezca como valor crítico de carga factorial de 0.7 (Chion \& Charles, 2016). En el factor de Educación y Capacidad, se eliminó la variable observable EET02. En el factor Normas Culturales y Sociales, se eliminó la variable observable CSN05. En la dimensión de Motivación de la Capacidad Emprendedora, se eliminaron las variables observables M03 y M04.

Por medio de la eliminación de las variables observables con bajas cargas en sus respectivos factores, se mejoró la consistencia interna de los factores. Además, se obtuvo aceptables de varianzas extraídas medias, con valores mayores al nivel crítico de 0.5 . Con ello, se asegura que los constructos son confiables para el estudio en de capacidad emprendedora en el contexto de graduados de la carrera de emprendimiento.

Tabla 2

Validez Convergente Modelo de Medición Ajustado

\begin{tabular}{|c|c|c|c|c|}
\hline & Alfa de Cronbach & rho_A & $\begin{array}{l}\text { Fiabilidad } \\
\text { compuesta }\end{array}$ & $\begin{array}{c}\text { Varianza extraída } \\
\text { media (AVE) }\end{array}$ \\
\hline Capacidad Emprendedora & 0.880 & 0.896 & 0.911 & 0.566 \\
\hline Educación y Capacitación & 0.873 & 0.874 & 0.908 & 0.664 \\
\hline Habilidades & 0.903 & 0.904 & 0.928 & 0.721 \\
\hline Motivación & 0.788 & 0.797 & 0.877 & 0.704 \\
\hline Normas culturales y sociales & 0.827 & 0.832 & 0.886 & 0.660 \\
\hline Transferencia de I+D & 0.889 & 0.895 & 0.916 & 0.646 \\
\hline
\end{tabular}

Con relación a la validez discriminante, se realizó el análisis por medio del criterio de Fornell y Larker, confirmando que los constructos refinados para el caso de capacidad emprendedora, son distintas según estándares estadísticos. De esta manera, se realizó el Análisis Factorial Confirmatorio, refinando constructos obtenido constructos confiables que permitan obtener conclusiones válidas en el contraste de hipótesis del modelo estructural. Previo al contraste de las hipótesis del modelo estructural, se evaluó la colinealidad entre los factores del modelo por medio del valor de inflación de varianza. Según los resultados obtenidos, nos existe problema de colinealidad entre los factores del modelo estructural, al tener valores de factor de inflación de varianza menor a cinco (Hair et al., 2014). 
Tabla 3

Validez Discriminante del Modelo Ajustado - Criterio Fornell y Larker

\begin{tabular}{lcccccc}
\hline & $\begin{array}{c}\text { Capacidad Em- } \\
\text { prendedora }\end{array}$ & $\begin{array}{c}\text { Educación y } \\
\text { Capacitación }\end{array}$ & Habilidades & Motivación & $\begin{array}{c}\text { Normas } \\
\text { culturales y } \\
\text { sociales }\end{array}$ & $\begin{array}{c}\text { Transfe- } \\
\text { rencia de } \\
\text { I+D }\end{array}$ \\
\hline $\begin{array}{l}\text { Capacidad Em- } \\
\text { prendedora }\end{array}$ & 0.752 & & & & \\
$\begin{array}{l}\text { Educación } \\
\text { Capacitación }\end{array}$ & 0.456 & 0.815 & & & \\
$\begin{array}{l}\text { Habilidades } \\
\text { Motivación }\end{array}$ & 0.954 & 0.453 & 0.849 & & \\
$\begin{array}{l}\text { Normas cultu- } \\
\text { rales y sociales }\end{array}$ & 0.769 & 0.308 & 0.542 & 0.839 & & \\
$\begin{array}{l}\text { Transferencia } \\
\text { de I+D }\end{array}$ & 0.525 & 0.614 & 0.718 & 0.383 & 0.812 & \\
\hline
\end{tabular}

Tabla 4

Hipótesis del Modelo Estructural Ajustado - PLS-SEM

\begin{tabular}{cccc}
\hline \multicolumn{3}{c}{ Hipótesis } & Coeficientes de Ruta \\
\hline $\mathrm{H}_{1}$ & EET $\rightarrow$ Capacidad para Crear un nuevo Negocio & 0.059 & Se rechaza \\
$\mathrm{H}_{2}$ & EET $\rightarrow$ CSN & 0.614 & Se acepta \\
$\mathrm{H}_{3}$ & $\mathrm{RDT} \rightarrow$ Capacidad para Crear un Nuevo Negocio & -0.003 & Se rechaza \\
$\mathrm{H}_{4}$ & $\mathrm{CSN} \rightarrow$ Capacidad para Crear un Nuevo Negocio & 0.650 & Se acepta \\
\hline
\end{tabular}

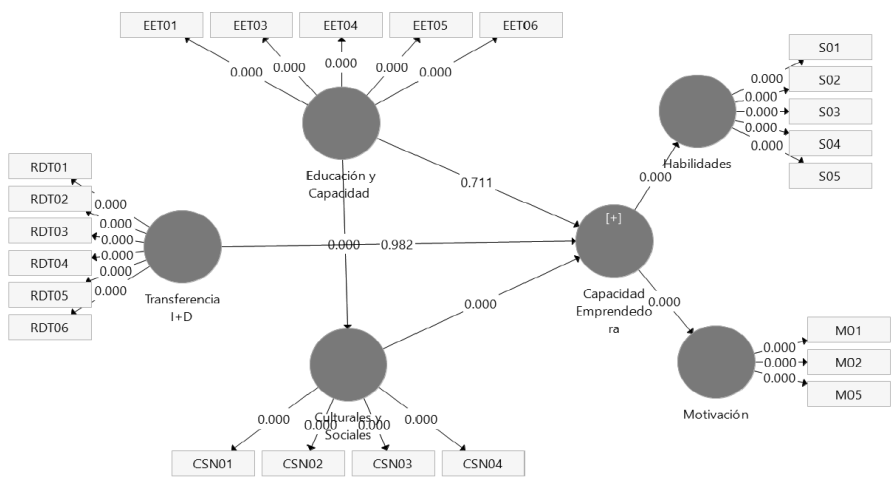

Figura 3. Modelo Estimado por Bootstrapping 
Para contrastar las hipótesis de una manera más objetiva, se sugiere estimar el modelo estructural por medio de bootstrapping con cinco mil sub muestras (Kwong \& Kay, 2013). Por medio de bootstrapping se puede contrastar las hipótesis por medio de la evaluación de los valores p con un nivel de significancia de 0.05 , el cual es nivel comúnmente utilizado en estudios de ciencias sociales. Por medio del procedimiento de bootstrapping con cinco mil submuestras, se confirma que las hipótesis 2 y la hipótesis 4 se aceptan con un nivel de significancia de 0.05 .

$$
\begin{aligned}
& \mathrm{H}_{2}: \mathrm{EET} \rightarrow \mathrm{CSN} \\
& \mathrm{H}_{4}: \mathrm{CSN} \rightarrow \text { Capacidad para Crear un Nuevo Negocio }
\end{aligned}
$$

Con ello, solo el factor de normas culturales y sociales tiene una influencia directa, positiva y significativa en la capacidad para crear un nuevo negocio. En la presente investigación, se demuestra que la educación y capacidad de emprendimiento y la transferencia de investigación y desarrollo no tienen una influencia directa y significativa en la capacidad de crear un nuevo negocio. Sin embargo, se demuestra que el factor de Educación y Capacidad en Emprendimiento tiene una influencia indirecta en la capacidad para la crear un nuevo negocio, esta relación es significativa si es mediada por las normas culturales y sociales.

Tabla 5

Hipótesis del Modelo Estructural Ajustado - PLS-SEM

\begin{tabular}{clcccc}
\hline \multicolumn{1}{c}{ Hipótesis } & Coeficientes & T Statistics & P value & \\
\hline $\mathrm{H}_{1}: \quad \begin{array}{l}\text { EET } \rightarrow \text { Capacidad para Crear un } \\
\text { nuevo Negocio }\end{array}$ & 0.059 & 0.371 & 0.711 & Se rechaza \\
$\mathrm{H}_{2}:$ & EET $\rightarrow$ CSN & 0.614 & 8.137 & 0.000 & Se acepta \\
$\quad$ RDT $\rightarrow$ Capacidad para Crear & -0.003 & .022 & 0.982 & Se rechaza \\
$\mathrm{H}_{3}: \quad \begin{array}{l}\text { un Nuevo Negocio } \\
\quad \text { CSN } \rightarrow \text { Capacidad para Crear un } \\
\mathrm{H}_{4}:\end{array}$ & 0.650 & 6.249 & 0.000 & Se acepta \\
\hline
\end{tabular}

Nota. Estimación con cinco mil submuestras

Para la evaluación del modelo ajustado de ecuaciones estructural por medio de la estimación por medio de mínimo cuadrados parciales, se evaluó la capacidad predictiva del modelo por medio del coeficiente de determinación y del efecto tamaño . Por medio de la estimación del modelo ajustado, con constructos refinados a través de la eliminación de las variables observables con bajas cargas factoriales, se pudo mejorar el coeficiente de determinación de 
0.442 a 0.447 . Aunque no existe un valor crítico para el en estudios de ciencias sociales, se considera que 0.442 es un valor aceptable con lo cual el $44.2 \%$ de la variación de la capacidad del crear un nuevo negocio es explicado por las variables exógenas del modelo.

Por otro lado, por medio de la evaluación de la capacidad predictiva del modelo por medio del efecto tamaño, se encontró que los factores Educación y Capacidad en Emprendimiento y el factor Trasferencia de Investigación y Desarrollo, no tienen capacidad predictiva de manera directa en la capacidad para crear un nuevo negocio. Por otro lado, el factor Normas Culturales y Sociales tiene una capacidad predictiva media en la capacidad para crear un nuevo negocio. Para complementar la evaluación de la calidad del modelo estimado, se analizó la relevancia de predictiva por medio valor de Stone-Geisser que puede ser obtenido por el procedimiento de Blidfolding, en el cual se indica la relevancia predictiva de un constructo exógeno en una variable latente endógena (Kwong \& Kay, 2013). Capacidad para crear un nuevo negocio $=0.242$

Se puede concluir que el modelo estructural propuesto tiene una capacidad predictiva media según el criterio de Hair et al. (2014). Para profundizar en los resultados, se evaluó la heterogeneidad del modelo por medio de la inclusión de la edad como variable moderado (Wong, 2016). Con ello, se buscó identificar si para ciertos rangos de edad los factores Educación y Capacidad en Emprendimiento y el factor Transferencia en Investigación y Desarrollo tienen una relación significativa directa en la Capacidad para Crear un Nuevo Emprendimiento.

Por medio de la estimación del efecto moderación de la edad, por medio del cálculo del producto, utilizando el algoritmo PLS, se encontró que la edad no modera significativamente la relación entre los factores independientes en la Capacidad para Crear un Nuevo Negocio. Los valores de coeficiente de ruta estandarizados para los efectos moderadores fueron menores a 0.02, cifra significativamente menor al valor crítico sugerido por Ringle et al. (2012). Además, se encontró que la edad tampoco tiene un efecto significativo directo en Capacidad para Crear un Nuevo Negocio.

Respecto al efecto moderador del sexo, se encontró que solo el sexo no modera de manera significativa la relación entre los factores independientes y la Capacidad para Crear un Nuevo Negocio. Se obtuvo un coeficiente de ruta estandarizado de 0.136 , por medio de la estimación por algoritmo PLS, en cual se considera que estaría próximo al valor de aceptación. Para confirmar de manera más objetiva este resultado, se estimó el modelo por bootstrapping con cinco mil sub muestras para el contraste de la hipótesis de efecto moderación por medio de valor p con un 0.05 de nivel de significancia. Por medio de la estimación por bootstrapping con cinco mil submuestras, se confirma el efecto moderador no significativo del sexo entre los factores independientes y la Capacidad para Crear un Nuevo Negocio 


\section{Discusión}

La cultura es un factor determinante en la forma en que los individuos afrontan las limitaciones para generar soluciones a corto plazo y la forma de enfrentar las limitaciones formales que las normas sociales pueden llegar a establecer en la eficacia de los empleados en el desarrollo empresarial a largo plazo. Los seres humanos construyen "modelos mentales" para intentar explicar e interpretar la realidad que los rodea y el ámbito empresarial no es la excepción, de acuerdo a lo expuesto en el enfoque teórico sociocultural que estudia los factores que inciden en la actividad emprendedora. (Díaz-Casero et al., 2005). Uno de los aspectos que no se puede quedar aislado en el estudio del emprendimiento es la forma de reaccionar del individuo condicionado por el entorno ya que las normas sociales y la cultural se ponen de manifiesto cuando se trata de analizar el crecimiento económico, la innovación de las empresas y la creación de empleo (Díaz-Casero, Hernández-Mogollón, Sánchez, \& Postigo, 2010).

Existen diferentes puntos de vista, creencias y motivaciones que provocan una duda interna al momento de tomar acción basada en el espíritu empresarial a nivel internacional. Los avances adicionales se ven obstaculizados por la falta de escalas válidas para medir las identidades sociales de los fundadores. Dado esto, se debe buscar una mejor compresión sobre emprendedores, basándose en vivencias y ejemplos enfocados a la teoría de la identidad social. (Sieger, Gruber, Fauchart, \& Zellweger, 2016). Los educadores en el campo universitario tienen la misión con el tiempo de acoplarse a los cambios y demandas de los estudiantes. Se debe buscar los intereses de ambos en todo momento reformando programas y a la vez economías locales. Con herramientas adecuadas que brinde la universidad permitirá abrir la mentalidad de los estudiantes universitarios y del mismo modo formar profesionales desde un punto de vista más innovador y empresarial (Sotirakou, 2016).

La capacidad emprendedora, conocida en inglés como entrepreneurial capacity (EC), es una herramienta que ayuda al individuo a descubrir las oportunidades del mercado antes de comprometer sus recursos y exponerse al riesgo de crear una empresa. (Clarysse et al., 2011) Estos autores citando a Baron y Ensley (2006) explicaron que identificar las oportunidades es una de las características que mejor definen al emprendedor y le permiten desarrollar sus habilidades para tener éxito en su emprendimiento. El reconocimiento de oportunidades está conectado con la capacidad emprendedora ya que el emprendedor que quiere resultados deberá analizar bien su entorno (Clarysse et al., 2011). No obstante, para estos autores la habilidad de reconocer las oportunidades presentes en el mercado no es una destreza exclusiva de ciertos emprendedores sino que más bien debería ser una habilidad requerida como condición inicial para aquellos que aspiran a emprender.

Para profundizar en el análisis de los resultados de la estimación del modelo, se realizó un análisis de heterogeneidad para determinar si las hipótesis logran ser aceptadas por 
diferencias en edades y en el sexo de los encuestados. De esta forma se encontró que para diferentes rangos de edades, para hombre y para mujeres, la educación y la transferencia de investigación - desarrollo no influencian la capacidad emprendedora de los graduados de la carrera de emprendimiento de la Universidad Católica de Santiago de Guayaquil y el Centro de Emprendedores de la Escuela Superior Politécnica del Litoral.

Recientes investigaciones han aportado de manera específica en cuanto a la variable latente entorno con nuevo conocimiento con respecto a los factores que inciden en la creación de un nuevo negocio. Para Díaz-Casero et al. (2012) los factores ambientales o del entorno que influyen en la creación de un nuevo negocio son: (a) la educación y capacitación empresarial, (b) la transferencia de la investigación y desarrollo; y (c) las normas culturales y sociales. Sus hallazgos demostraron el rol excepcional de normas culturales y sociales en la capacidad de generar nuevos negocios. Sin embargo, estos autores concluyeron que la educación y capacitación en la creación de nuevos negocios no influye significativamente en dicha creación (no está asociada de manera positiva con la creación de nuevos negocios) sino que más bien es un facilitador indirecto a través de la normas culturales y sociales de la población.

\section{Conclusiones}

El presente estudio buscó determinar los factores que influencian a la creación de unidades de negocios. Para ello, se tomó como unidad de análisis a una muestra de estudiantes graduados de la carrera de emprendimiento de la Universidad Católica Santiago de Guayaquil el Centro de Emprendedores de la Escuela Superior Politécnica del Litoral. Para el levantamiento de los datos se utilizó el cuestionario validado y para la evaluación de la estructura de los factores del modelo y las hipótesis del modelo estructural, se estimaron modelos de ecuaciones estructurales por medio del programa SMART PLS versión 3.

Luego de confirmar la confiabilidad de los factores por medio del análisis de consistencia interna, validez discriminante y convergente se estimó el modelo estructural por medio de PLS-SEM. Por medio de la estimación de coeficientes de ruta del modelo estructural, se logró aceptar dos de las cuatro hipótesis:

$$
\begin{aligned}
& \mathrm{H}_{2}: \mathrm{EET} \rightarrow \mathrm{CSN} \\
& \mathrm{H}_{4}: \mathrm{CSN} \rightarrow \text { Capacidad para Crear un Nuevo Negocio }
\end{aligned}
$$

Solo el factor de normas culturales y sociales tiene una influencia directa, positiva y significativa en la capacidad para crear un nuevo negocio. Mientras que, la educación y capacidad de emprendimiento y la transferencia de investigación más desarrollo no tiene una influencia directa y significativa en la capacidad de crear un nuevo negocio. Sin embargo, se demuestra que el factor de Educación y Capacidad en Emprendimiento tiene una influencia indirecta en 
la capacidad para la crear un nuevo negocio, esta relación es significativa si es mediada por las normas culturales y sociales. Para profundizar en el análisis de los resultados de la estimación del modelo, se realizó un análisis de heterogeneidad para determinar si las hipótesis logran ser aceptadas por diferencias en edades y en el sexo de los encuestados. De esta forma se encontró que para diferentes rangos de edades, para hombre y para mujeres, la educación y la transferencia de investigación - desarrollo no influencian la capacidad emprendedora de los graduados de la carrera de emprendimiento de la Universidad Católica de Santiago de Guayaquil y el Centro de Emprendedores de la Escuela Superior Politécnica del Litoral.

En vista de la importancia que implica la creación de nuevas empresas, los gobiernos están estableciendo políticas públicas que permitan incrementar la cantidad de emprendimientos (Acs, Åstebro, Audretsch, \& Robinson, 2016), ya que además de ser beneficioso para el desarrollo y crecimiento económico, se ha comprobado que ayudan en la reducción de la pobreza y mejoran la efectividad y sostenibilidad de la ayuda económica provista por los organismos internacionales (Naudé, 2013). Los determinantes que permiten la creación de nuevas empresas han sido menos estudiados en los países en desarrollo que en los países desarrollados. La literatura nos revela que los investigadores han considerado el entorno institucional (estabilidad macroeconómica, políticas públicas y conocimiento) y ciertos factores de oferta (ingresos y financiamiento) y demanda (estructura industrial e ingresos) como los factores diferenciales del emprendimiento en los países desarrollados, sin embargo considerando otros factores en una serie de estudios comparativos entre países, se concluye que el bajo nivel de emprendimiento en los países en desarrollo se debe a una mayor burocracia y peores estructuras de gobernanza (Calá, Arauzo-Carod, \& Manjón-Antolín, 2015; Sieger et al., 2016).

Entre las principales limitaciones del estudio están el tamaño de la muestra y que los datos fueron obtenidos en un único momento en el tiempo, con lo cual solo se puede llegar a conclusiones de asociación de variables. Se sugiere que en futuras investigaciones se amplíe el tamaño de muestra por medio de la inclusión de estudiantes graduados de carreras de emprendimiento de otras universidades. Otro aspecto que pretende abarcar la presente investigación es proponer mejoras a la oferta educativa de la población consultada como respuesta a las limitaciones que manifestaron al momento de afrontar el reto de desarrollar nuevas unidades de negocio o los llamados emprendimientos de oportunidad; uno de los resultados obtenidos en la investigación estableció que la cultura es un factor determinante en la forma en que los individuos afrontan las limitaciones para generar soluciones a corto plazo y la forma de enfrentar dichas limitaciones para desarrollo empresarial a largo plazo.

El aprendizaje que se brinda en las universidades tiene un efecto tanto positivo como negativo en los estudiantes. Es decir, si se lo da de forma correcta ayudará a mejorar las capacidades y habilidades de cada uno de ellos, pero si no se lo realiza de la forma o con el enfoque correcto generará miedos viendo este modelo de estudio como un reto poco productivo 
(Zhang, Duysters, \& Cloodt, 2013). Se debe de manejar de manera más realista, entrando más a profundidad siendo imparciales en todo momento y teniendo como objetivo principal intenciones más comprometidas y realistas. (Sieger, Fueglistaller, \& Zellweger, 2016). Una forma positiva de incentivar la formación emprendedora en universidades y la participación de estudiantes es creando proyectos o convenios con empresas. De esta forma, los estudiantes podrán poner en práctica lo que se aprende en clases e involucrarse de forma activa y dinámica en el entorno cotidiano de una empresa o un emprendedor. El programa diseñado no solo se basa en aspectos mencionados anteriormente sino involucrar a la tecnología como un aspecto fundamental (Klofsten, 2000)including professorships in entrepreneurialism at universities, new courses and training programmes, while firms such as Ericsson, Telia and Saab have established "innovation units" to stimulate new ideas and utilize the energy and ideas of their employees. The purpose of this article is to describe and analyse such an initiative: the Entrepreneurship and New Business Development Programme (the ENP-programme.

Por otra parte, si la cultura es un factor determinante, entonces se usará este factor para generar unidades de emprendimiento cuando el estudiante termine la carrera. Para ello, se sugiere que los estudiantes de las carreras empresariales durante los primeros dos semestres de formación puedan participar en eventos culturales, como talleres, encuentros de emprendedores de distintas partes del país, portafolio de proyectos de emprendimiento en negocio de diferentes zonas del Ecuador, entre otros. Asimismo, que se transforme el aula de clase en laboratorios, en donde sea posible observar las prácticas gerenciales para establecer la evolución de la identidad de una organización a través del desempeño de los estudiantes de carreras empresariales o de negocios (emprendedores o no) de las distintas culturas existentes (ciudades), por ello es necesario ampliar la investigación a otros ámbitos del país donde la cultura tiene un papel protagónico en la formación de nuevas unidades de negocios.

Otra acción que se puede proponer para el fortalecimiento de la formación del emprendedor y futuro empresario son los sistemas de acciones que les permitan comprender las necesidades de la sociedad. Asimismo, debe crearse espacios que desarrollen las capacidades emprendedoras a través de la visualización de oportunidades, generar debate científico, con expertos conferencistas en el área para coadyuvar al desarrollo de una nueva generación de emprendedores ecuatorianos, donde se comparta experiencias de emprendimiento de acuerdo a la idiosincrasia y cultura de cada ciudad del Ecuador.

Se propone que se inserte en la oferta académica la tecnología de manera que se provea al estudiante o emprendedor en formación de una amplia información sobre los principales problemas que enfrenta la profesión y la situación socioeconómica y política, por distintas regiones y ciudades del país, creación de bancos de problemas que afrontan los emprendedores, publicaciones acerca de los proyectos de vida como futuros emprendedores. Todo este tipo de innovaciones hacen evidente que se debe investigar con urgencia los requerimientos 
educativos de los profesionales del emprendimiento o carreras de negocios existentes en el Ecuador para conocer si comparten las necesidades antes mencionadas.

\section{Referencias}

Acs, Z., Åstebro, T., Audretsch, D., \& Robinson, D. T. (2016). Public policy to promote entrepreneurship. A call to arms. Small Business Economics, 47(1), 35-51. https://doi.org/10.1007/s11187-016-9712-2

Ajzen, I. (1991). The Theory of Planned Behavior on Organizational Behavior and decision processes. (pp 179211) Chicago, IL: Dorsey Press. Vol 50.

Babina, T., \& Howell, S. (2018). Entrepreneurial Spillovers from Corporate R\&D (N. ${ }^{\circ}$ w25360; p. w25360). https:// doi.org/10.3386/w25360

Baron, R., \& Ensley, M. (2006). Opportunity recognition as the detection of meaningful patterns: Evidence from comparisons of novice and experienced entrepreneurs. Management science, 52(9), 1331-1344.

Brown, T. (2015). Confirmatory factor analysis for applied research (Second ed.). New York: The Guilford Press.

Calá, C. D., Arauzo-Carod, J.-M., \& Manjón-Antolín, M. (2015). The determinants of entrepreneurship in developing countries. Recuperado de http:/www.urv.cat/creip/media/upload//arxius/wp/WP2015/DT.01-2015100_Cala_Arauzo_Manjon.pdf

Clarysse, B., Tartari, V., \& Salter, A. (2011). The impact of entrepreneurial capacity, experience and organizational support on academic entrepreneurship. Research Policy, 40(8), 1084-1093. https://doi.org/10.1016/j.respol.2011.05.010

Cole, A. (1965). An approach to the study of entrepreneurship: A tribute to Edwin F. Gay. En H. G. J. Aitken (Ed.), Explorations in enterprise (pp. 30-44). Cambridge, MA: Harvard University Press.

Cooper, S., \& Park, J. (2008). The Impact of 'Incubator' Organizations on Opportunity Recognition and Technology Innovation in New, Entrepreneurial High-technology Ventures. International Small Business Journal, 26(1), 27-56. https://doi.org/10.1177/0266242607084658

Chion, S., \& Charles, V. (2016). Analítica de datos para la modelación estructural. Lima: Pearson.

DeTienne, D., \& Chandler, G. (2004). Opportunity Identification and Its Role in the Entrepreneurial Classroom: A Pedagogical Approach and Empirical Test. Academy of Management Learning \& Education, 3(3), $242-257$. https://doi.org/10.5465/AMLE.2004.14242103

Díaz-Casero, J.,Hernández-Mogollón,R., \& Roldán,J. (2012).AStructural Model of the Antecedents to Entrepreneurial Capacity. International Small Business Journal, 30(8), 850-872. https://doi.org/10.1177/0266242610385263

Díaz-Casero, J., Hernández, R., Sánchez, M., \& Postigo Jiménez, M. (2010). Actividad emprendedora y género: Un estudio comparativo. Revista Europea de Dirección y Economía de la Empresa, 19(2), 83-98.

Díaz-Casero, J., Urbano-Pulido, D., \& Hernández-Mogollón, R. (2005). Teoría económica institucional y creación de empresas. Investigaciones Europeas de Dirección y Economía de la Empresa, 209-230.

Franke, N., \& Lüthje, C. (2004). Entrepreneurial intentions of business students - A benchmarking study. International Journal of Innovation and Technology Management, 1(3), 269-288

Garson, D. (2016). Partial least squares: Regression \& structural equation models. Asheboro: Statistical Associates Blue Book Series.

Gartner, W. B. (1985). A conceptual framework for describing the phenomenon of new venture creation. Academy of Management Review, 10(4), 696-706. https://doi.org/10.5465/AMR.1985.4279094

George, G., \& Zahra, S. (2002). Culture and Its consequences for entrepreneurship. Entrepreneurship: Theory \& Practice, 26(4), 5-19.

Gibb, A., \& Hannon, P. (2006). Towards the entrepreneurial university. International Journal of Entrepreneurship Education, 5(1), 73-110. 
Hair, J., Hult, T., Ringle, C., \& Sarstedt, M. (2014). A premier on partial least squares structural equation modeling (PLS-SEM). Los Angeles: SAGE.

Harrington, D. (2009). Confirmatory factor analysis. New York: Oxford University Press.

Henry, C., Hill, F., \& Leitch, C. (2005). Entrepreneurship education and training: can entrepreneurship be taught? Part I. Education+Training, 47(2), 98-111.

Hernández, R., Fernández, C., \& Baptista, P. (2014). Metodología de la investigación (6ta edición). México: McGraw Hill.

Hofstede, G. (1980). Culture's Consequences: International Differences in Work-Related Values. Beverly Hills, Calif: Sage Publications.

Honig, B. (2004). Entrepreneurship education: Toward a model of contingency-based business planning. Academy of Management Learning \& Education, 3(3), 258-273. https://doi.org/10.5465/AMLE.2004.14242112

Hughes, M., Ireland, R. D., \& Morgan, R. E. (2007). Stimulating dynamic value: Social capital and business incubation as a pathway to competitive success. Long Range Planning, 40(2), 154-177

Klofsten, M. (2000). Training entrepreneurship at universities: a Swedish case. Journal of European Industrial Training, 24(6), 337-344.

Krishnan, Nvh, Sivramkrishna, S., \& Warrier, U. (2017). Entrepreneurial capacity creation through occupational shift and sustenance orientation of women micro entrepreneurs. International Journal of Management Studies, $4(1), 2231-2528$.

Kwong, K., \& Kay, W. (2013). Partial least squares structural equation modeling (PLS-SEM) techniques using SmartPLS. Marketing Bulletin, 24(1), 1-32.

Lenox, M., \& York, J. G. (2012). 'Environmental entrepreneurship'. In Bansal, P. and Hoffman, A. J.(Eds), The Oxford Handbook of Business and the Natural Environment. New York: Oxford University Press, 70-82.

Levie, J., \& Autio, E. (2008). A theoretical grounding and test of the GEM model. Small Business Economics, 31(3), 235-263. https://doi.org/10.1007/s11187-008-9136-8.

Matejun, M. (2016). Role of technology entrepreneurship in the development of innovativeness of small and medium-sized enterprises. Management, 20(1), 167-183. https://doi.org/10.1515/manment-2015-0032

Naudé, W. (2013). Entrepreneurship and Economic Development: Theory, Evidence and Policy (SSRN Scholarly Paper N. ${ }^{\circ}$ ID 2314802). Recuperado de Social Science Research Network website: https://papers.ssrn.com/ abstract $=2314802$

Nelson, R., \& Winter, S. (1982). An Evolutionary Theory of Economic Change. Cambridge, Mass.: The Belknap Press of Harvard Univ. Press.

Nielsen, S. L., \& Lassen, A. H. (2012). Identity in entrepreneurship effectuation theory: a supplementary framework. International Entrepreneurship and Management Journal, 8(3), 373-389.

Norton, S. (1988). Franchising, brand name capital, and the entrepreneurial capacity problem. Strategic Management Journal, 9(1), 105-114.

Panigrahi, A. (2015, enero 1). Entrepreneurship Education and Economic Development: An Indian Perspective.

Peterman, N., \& Kennedy, J. (2003). Enterprise education: Influencing students' perceptions of entrepreneurship. Entrepreneurship Theory and Practice, 28(2), 129-144. https://doi.org/10.1046/j.1540-6520.2003.00035.x

Parker, S. C., \& Belghitar, Y. (2006). What happens to nascent entrepreneurs? An econometric analysis of the PSED. Small Business Economics, 27(1), 81-101.

Politis, D., Winborg, J., \& Dahlstrand, Å. L. (2012). Exploring the resource logic of student entrepreneurs. International Small Business Journal, 30(6), 659-683.

Raposo, M., \& Paço, A. (2011). Entrepreneurship education: Relationship between education and entrepreneurial activity. Psicothema, 23(1), 453-457.

Reynolds, P., Bosma, N., Autio, E., Hunt, S., De Bono, N., Servais, I., .. Chin, N. (2005). Global Entrepreneurship Monitor: Data collection design and implementation 1998 - 2003. Small Business Economics, 24(3), 205-231. https://doi.org/10.1007/s11187-005-1980-1 
Ringle, C., Sarstedt, M., \& Straub, D. (2012). A critical look at the use of PLS-SEM in MIS Quarterly. MIS Quarterly, 36(1), 1-19.

Ringle, C., Sven, W., \& Becker, J.-M. (2015). Obtenido de Bönningstedt: SmartPLS: http://www.smartpls.com

Robinson, P. B., \& Sexton, E. A. (1994). The effect of education and experience on self-employment success. Journal of Business Venturing, 9(2), 141-156.

Rothaermel, F. T., Agung, S. D., \& Jiang, L. (2007). University entrepreneurship: a taxonomy of the literature. Industrial and Corporate Change, 16(4), 691-791.

Rovayo, G. (2013). El emprendimiento y la educación no siempre van en la misma dirección.

Schumpeter, J. (1934). The Theory of Economic Development: An Inquiry into Profits, Capital, Credit, Interest, and the Business Cycle. Harvard University Press.

Sesen, H. (2013). Personality or environment? A comprehensive study on the entrepreneurial intentions of university students. Education+ Training, 55(7), 624-640.

Shane, S. (2000). Prior knowledge and the discovery of entrepreneurial opportunities. Organization Science, 3(4), 448-452.

Shirokova, G., Osiyevskyy, O., \& Bogatyreva, K. (2016). Exploring the intention-behavior link in student entrepreneurship: Moderating effects of individual and environmental characteristics. European Management Journal, 34(4), 386-399.

Sieger, P. (2014). Student Entrepreneurship across the globe: a look at intention and activities. Global University Entrepreneurial spirit students' survey. Swiss Research Institute of Small Business and Entrepreneurship. University of St. Gallen

Sieger, P., Fueglistaller, U., \& Zellweger, T. (2016). Student entrepreneurship 2016: insights from 50 countries.

Sieger, P., Gruber, M., Fauchart, E., \& Zellweger, T. (2016). Measuring the social identity of entrepreneurs: Scale development and international validation. Journal of Business Venturing, 31(5), 542-572.

Smith, P., Peterson, M., \& Schwartz, S. (2002). Cultural values, sources of guidance, and their relevance to managerial behavior: A 47 - nation study. Journal of Cross-Cultural Psychology, 33(2), 188-208. https://doi. org/10.1177/0022022102033002005

Sotirakou, T. (2004). Coping with conflict within the entrepreneurial university: Threat or challenge for heads of departments in the UK higher education context. International Review of Administrative Sciences, 70(2), 345372.

Thornton, P., Ribeiro-Soriano, D., \& Urbano, D. (2011). Socio-cultural factors and entrepreneurial activity: An overview. International Small Business Journal: Researching Entrepreneurship, 29(2), 105-118. https://doi. org/10.1177/0266242610391930

Wong, K. (2016). Technical note: Mediation analysis, categorical moderation analysis, and higher-order constructs modeling in partial least squares structural equation modeling (PLS-SEM): A B2B example using SmartPLS. The Marketing Bulletin, 26(1), 1-23

Zhao, H., Seibert, S. E., \& Hills, G. E. (2005). The mediating role of self-efficacy in the development of entrepreneurial intentions. Journal of Applied Psychology, 90(6), 1265-1272.

Zhang, Y., Duysters, G., \& Cloodt, M. (2014). The role of entrepreneurship education as a predictor of university students' entrepreneurial intention. International Entrepreneurship and Management Journal, 10(3), 623-641. 